\title{
The oxygen abundance distribution in $\mathrm{M} 101$
}

\author{
L. S. Pilyugin \\ Main Astronomical Observatory of National Academy of Sciences of Ukraine, Goloseevo, 03680 Kiev-127, Ukraine \\ Received 15 March 2001 / Accepted 23 April 2001

\begin{abstract}
The well-observed spiral galaxy M 101 was considered. The radial distributions of oxygen abundances determined in three different ways (with the classic $T_{\mathrm{e}}$-method, with the $R_{23}$-method, and with the $P$-method) were compared. It was found that the parameters (the central oxygen abundance and the gradient) of the radial $(\mathrm{O} / \mathrm{H})_{P}$ abundance distribution are close to those of the $(\mathrm{O} / \mathrm{H})_{T_{\mathrm{e}}}$ abundance distribution. The parameters of the $(\mathrm{O} / \mathrm{H})_{R_{23}}$ abundance distribution differ significantly from those of the $(\mathrm{O} / \mathrm{H})_{T_{\mathrm{e}}}$ abundance distribution: the central $(\mathrm{O} / \mathrm{H})_{R_{23}}$ oxygen abundance is higher by around 0.4 dex and the gradient is steeper by a factor of around 1.5 as compared to those values in the $(\mathrm{O} / \mathrm{H})_{T_{\mathrm{e}}}$ abundance distribution. The dispersion in $(\mathrm{O} / \mathrm{H})_{P}$ abundance at fixed radius is rather small, $\sim 0.08 \mathrm{dex}$, and is equal to that in $(\mathrm{O} / \mathrm{H})_{T_{\mathrm{e}}}$ abundance. The dispersion in $(\mathrm{O} / \mathrm{H})_{R_{23}}$ abundance at fixed radius is appreciably larger, $\sim 0.16 \mathrm{dex}$, compared to that in $(\mathrm{O} / \mathrm{H})_{T_{\mathrm{e}}}$ abundance. It has been shown that the extra dispersion in $(\mathrm{O} / \mathrm{H})_{R_{23}}$ abundances is an artifact and reflects scatter in excitation parameter $P$ at fixed radius.
\end{abstract}

Key words. galaxies: abundances - galaxies: ISM - galaxies: spiral - galaxies: individual: M 101

\section{Introduction}

By now spectra have been obtained for hundreds of HII regions in spiral galaxies. Accurate oxygen abundances can be derived from measurement of temperature-sensitive line ratios, such as [OIII]4959, $5007 /[\mathrm{OIII}] 4363$. This method will be referred to as the $T_{\mathrm{e}}$-method. Unfortunately, in oxygen-rich $\mathrm{H}$ II regions the temperature-sensitive lines such as [OIII]4363 are too weak to be detected. For such H II regions, empirical abundance indicators based on more readily observable lines were suggested (Pagel et al. 1979; Alloin et al. 1979). The empirical oxygen abundance indicator $R_{23}=([\mathrm{OII}] 3727$, $3729+[\mathrm{OIII}] 4959,5007) / \mathrm{H}_{\beta}$, suggested by Pagel et al. (1979), has found widespread acceptance and use for the oxygen abundance determination in $\mathrm{H}$ II regions where the temperature-sensitive lines are undetectable. This method will be referred to as the $R_{23}$-method. Using the $R_{23^{-}}$ method, the characteristic oxygen abundances (the oxygen abundance at a predetermined galactocentric distance) and radial oxygen abundance gradients were obtained for a large sample of spiral galaxies (Vila-Costas \& Edmunds 1992; Zaritsky et al. 1994; van Zee et al. 1998, among others).

Hovewer, the basic problem whether $R_{23}$ is an accurate abundance indicator is open for discussion (Zaritsky 1992; Kinkel \& Rosa 1994, among others). It has been found

Send offprint requests to: L. S. Pilyugin, e-mail: pilyugin@mao.kiev.ua
(Pilyugin 2000, Paper I) that the error in the oxygen abundance derived with the $R_{23}$-method involves two parts: the first is a random error and the second is a systematic error depending on the excitation parameter. A new way of oxygen abundance determination in Hil regions $(P-$ method) was suggested (Paper I, Pilyugin 2001, Paper II). By comparing oxygen abundances in high-metallicity H II regions derived with the $T_{\mathrm{e}}$-method and those derived with the $P$-method, it was found that the precision of oxygen abundance determination with the $P$-method is comparable to that of the $T_{\mathrm{e}}-$ method (Paper II). It was shown that the $R_{23}$-method provides more or less realistic oxygen abundances in high-excitation H II regions, but yields overestimates in low-excitation ones. Taking into account this fact together with the fact known for a long time (Searle 1971; Smith 1975) that galaxies can show strong radial excitation gradients, in the sense that only low-excitation $\mathrm{H}$ II regions populate the central parts of some galaxies, one can expect that the central oxygen abundances and gradient slopes based on the $(\mathrm{O} / \mathrm{H})_{R_{23}}$ data can be appreciably overestimated.

This speculation can have far-reaching implications (the empirical estimation of the oxygen yield, correlations of the behaviour of oxygen abundances with other properties of spiral galaxies, etc.). This speculation can be verified by comparison of the radial $(\mathrm{O} / \mathrm{H})_{R_{23}}$ abundance distribution with the radial $(\mathrm{O} / \mathrm{H})_{T_{\mathrm{e}}}$ abundance distribution. The well-observed spiral galaxy M 101 provides such possibility. 
The comparison between radial distributions of $(\mathrm{O} / \mathrm{H})_{T_{\mathrm{e}}},(\mathrm{O} / \mathrm{H})_{P}$, and $(\mathrm{O} / \mathrm{H})_{R_{23}}$ abundances across the disk of M 101 aiming to test the credibility of the $(\mathrm{O} / \mathrm{H})_{P}$ and $(\mathrm{O} / \mathrm{H})_{R_{23}}$ abundances is a goal of the present study.

\section{The oxygen abundance distribution in $M 101$}

\subsection{General comments}

Despite the fact that the spectroscopic data with detections of diagnostic emission lines in $\mathrm{H}$ II regions makes it possible to determine the accurate oxygen abundance $(\mathrm{O} / \mathrm{H})_{T_{\mathrm{e}}}$, the oxygen abundances in the same $\mathrm{HII}$ region with measured line ratios $[\mathrm{OIII}] \lambda \lambda 4959,5007 / \lambda 4363$ derived in different works can differ for three reasons: atomic data adopted, interpretation of the temperature structure (single characteristic $T_{\mathrm{e}}$, two-zone model for $T_{\mathrm{e}}$, model with small-scale temperature fluctuations) and errors in the line intensity measurements. Therefore the compilation of $\mathrm{HII}$ regions with original oxygen abundance determinations through the $T_{\mathrm{e}}$-method from different works carried out over more than twenty years is not a set of homogeneous determinations. Accordingly, the available published spectra of $\mathrm{H}$ II regions with measured line ratios $[\mathrm{OIII}] \lambda \lambda 4959,5007 / \lambda 4363$ have been reanalysed to produce a homogeneous set. Two-zone models of $\mathrm{H}$ II regions with the algorithm for oxygen abundance determination from Pagel et al. (1992) and $T_{\mathrm{e}}([\mathrm{OII}])-T_{\mathrm{e}}([\mathrm{OIII}])$ relation from Garnett (1992) were adopted here.

The determination of the $(\mathrm{O} / \mathrm{H})_{P}$ oxygen abundances in high-metallicity $\mathrm{H}$ II regions has been considered in Paper II. The following expression has been suggested

$12+\log (\mathrm{O} / \mathrm{H})_{P}=\frac{R_{23}+54.2+59.45 P+7.31 P^{2}}{6.07+6.71 P+0.37 P^{2}+0.243 R_{23}}$,

where $R_{23}=R_{2}+R_{3}, R_{2}=I_{[\mathrm{OII}] \lambda 3727+\lambda 3729} / I_{\mathrm{H} \beta}, R_{3}=$

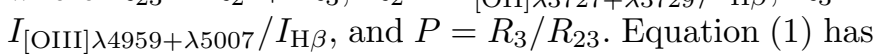
been used here for the determination of the $(\mathrm{O} / \mathrm{H})_{P}$ oxygen abundances.

Several workers have suggested calibrations of the $R_{23}$ in terms of the oxygen abundance (Edmunds \& Pagel 1984; McCall et al. 1985; Dopita \& Evans 1986; Zaritsky et al. 1994, among others). The most frequently used calibration after Edmunds \& Pagel (1984) has been adopted here for the determination of the $(\mathrm{O} / \mathrm{H})_{R_{23}}$ oxygen abundances.

\subsection{Radial oxygen abundance gradient}

The nearby Sc galaxy M $101=$ NGC 5457 has long served as the prototype system for studying the radial oxygen abundance gradients in disks. Spectroscopic observations of HII regions in M 101 have been carried out by many investigators (Smith 1975; Shields \& Searle 1978; Rayo et al. 1982; McCall et al. 1985; Torres-Peimbert et al. 1989; Garnett \& Kennicutt 1994; Kinkel \& Rosa 1994; Kennicutt \& Garnett 1996; van Zee et al. 1998; Garnett et al. 1999).
Table 1. Oxygen abundances in H II regions of spiral galaxy M 101. The name of Hil region is reported in Col. 1. The oxygen abundance computed here in common way (through the $T_{\mathrm{e}}$-method) is reported in Col. 2. The oxygen abundances in $\mathrm{H}$ II region Searle 5 derived with $T_{\mathrm{e}}\left([\mathrm{OII})\right.$ and $T_{\mathrm{e}}([\mathrm{NII})$ are labeled with letters $a$ and $b$ respectively. Source of line intensities measurements is given in Col. 3. The fractional radius $\rho$, normalized to the disk isophotal radius, is listed in Col. 4.

\begin{tabular}{lclc}
\hline \hline H II region & $12+\log (\mathrm{O} / \mathrm{H})_{T_{\mathrm{e}}}$ & reference & $\rho$ \\
\hline Searle 5 & $8.55^{a}$ & KR94 & 0.22 \\
$+252-107$ & $8.77^{b}$ & KR94 & \\
NGC 5461 & 8.55 & M85 & 0.33 \\
& 8.40 & S75 & 0.34 \\
NGC 5455 & 8.50 & R82 & \\
& 8.42 & T89 & \\
$-347+276$ & 8.40 & S75 & 0.48 \\
$-459-053$ & 8.43 & SS78 & \\
NGC 5447 & 8.45 & T89 & \\
Searle 12 & 8.34 & vZ98 & 0.54 \\
$-398-436$ & 8.19 & S75 & 0.55 \\
NGC 5471 & 8.06 & vZ98 & 0.55 \\
& 7.99 & S75 & 0.68 \\
& 8.17 & SS78 & \\
& 8.18 & R82 & \\
H681 & 8.11 & T89 & \\
$+010+885$ & 8.10 & G99 & \\
\hline \hline
\end{tabular}

List of references:

G99 - Garnett, et al. (1999); GK94 - Garnett \& Kennicutt (1994); KR94 - Kinkel \& Rosa (1994); M85 - McCall, et al. (1985); R82 - Rayo, et al. (1982); S75 - Smith (1975); SS78 - Shields \& Searle (1978); T89 - Torres-Peimbert, et al. (1989); vZ98 - van Zee, et al. (1998).

The H II regions of M 101 with measured temperaturesensitive line ratios are listed in Table 1 . The name of $\mathrm{H}$ II region is reported in Col. 1. The oxygen abundance $(\mathrm{O} / \mathrm{H})_{T_{\mathrm{e}}}$ recomputed here is reported in Col. 2. Source of line intensities measurements is given in Col. 3. The fractional radius $\rho$, normalized to the disk isophotal radius $\rho_{0}$, is listed in Col. 4 . The galactocentric distances were taken from Kennicutt \& Garnett (1996). The electron temperatures $T_{\mathrm{e}}([\mathrm{OIII}])$ in $\mathrm{H}$ II regions (with one exception, H II region Searle 5) have been determined from the measurements of $[\mathrm{OIII}] \lambda \lambda 4959,5007 / \lambda 4363$ line ratios, and the electron temperatures $T_{\mathrm{e}}([\mathrm{OII}])$ in $\mathrm{H}$ II regions have been derived from the $T_{\mathrm{e}}([\mathrm{OII}])-T_{\mathrm{e}}([\mathrm{OIII}])$ relation of Garnett (1992). In the case of H II region Searle 5 the electron temperature $T_{\mathrm{e}}([\mathrm{OIII}])$ cannot be directly determined from observational data since the measurement of $[\mathrm{OIII}] \lambda \lambda 4959,5007 / \lambda 4363$ line ratio is not available. 
Instead the temperature-sensitive lines [OII $\lambda \lambda \lambda 7320,7330$ and $[\mathrm{NII}] \lambda 5755$ were detected in deep spectrophotometry of Hil region Searle 5 (Kinkel \& Rosa 1994). Then the electron temperature $T_{\mathrm{e}}([\mathrm{OIII}])$ in $\mathrm{H}$ II region Searle 5 has been derived from the $T_{\mathrm{e}}([\mathrm{OII}])-T_{\mathrm{e}}([\mathrm{OIII}])$ relation of Garnett (1992) using the value of $T_{\mathrm{e}}([\mathrm{OII}])$ reported by Kinkel \& Rosa (1994). The oxygen abundance in H II region Searle 5 based on the $T_{\mathrm{e}}$ ([OII) is labeled with letter $a$ in Table 1 . The other value of oxygen abundance in $\mathrm{H}$ II region Searle 5 has been computed using the value of $T_{\mathrm{e}}([\mathrm{NII})$ from Kinkel \& Rosa (1994) and assuming $T_{\mathrm{e}}([\mathrm{OII}])=T_{\mathrm{e}}([\mathrm{NII}])$. The oxygen abundance in $\mathrm{H} \mathrm{II}$ region Searle 5 based on the $T_{\mathrm{e}}$ ([NII) is labeled with letter $b$ in Table 1.

In Fig. 1a we show oxygen abundances $(\mathrm{O} / \mathrm{H})_{T_{\mathrm{e}}}$ (the filled circles) for $\mathrm{H}$ II regions from Table 1 as a function of galactocentric distance. As seen in Fig. 1a the $(\mathrm{O} / \mathrm{H})_{T_{\mathrm{e}}}$ data is sufficient in quantity and quality for an accurate determination of the value of the oxygen abundance gradient within the M 101. It is evident from Fig. 1a that the radial distribution of oxygen abundance within the disk of M 101 can be reproduced by a single line. The best fit to the $(\mathrm{O} / \mathrm{H})_{T_{\mathrm{e}}}$ data is

$$
12+\log (\mathrm{O} / \mathrm{H})_{T_{\mathrm{e}}}=8.81( \pm 0.08)-0.028( \pm 0.005) R_{\mathrm{G}},
$$

where $R_{\mathrm{G}}$ is in kpc. The $(\mathrm{O} / \mathrm{H})_{T_{\mathrm{e}}}-R_{\mathrm{G}}$ relation is presented by the solid line in Fig. 1. The uncertainty of the value of central oxygen abundance is specified by the mean deviation of positions of $\mathrm{HI}$ regions from the $\mathrm{O} / \mathrm{H}-R_{\mathrm{G}}$ relation. The maximum value of the error in the slope expressed in units of $\operatorname{dex} / \rho_{0}$ (where $\rho_{0}$ is the isophotal radius) can be adopted equal to twice value of the mean deviation. The error in the slope expressed in units of dex $/ \mathrm{kpc}$ can be estimated through the division of the error in the slope expressed in units of $\operatorname{dex} / \rho_{0}$ by isophotal radius in $\mathrm{kpc}$, that gives $-0.028 \pm 0.005 \mathrm{dex} / \mathrm{kpc}$.

Figure $1 \mathrm{~b}$ shows the radial $(\mathrm{O} / \mathrm{H})_{P}$ abundance distribution (the open circles) for $\mathrm{H}$ II regions from Kennicutt \& Garnett (1996). The oxygen abundances in H II regions with galactocentric distances larger than $\sim 22 \mathrm{kpc}$ are expected (Eq. (2)) to be less than $12+\log \mathrm{O} / \mathrm{H}=8.2$, i.e they do not belong to the upper branch of the $R_{23}-\mathrm{O} / \mathrm{H}$ diagram. Since the Eq. (1) can be used for oxygen abundance determination in $\mathrm{H}$ II regions of the upper branch of the $R_{23}-\mathrm{O} / \mathrm{H}$ diagram only, the $\mathrm{H}$ II regions of M 101 with galactocentric distances larger than $22 \mathrm{kpc}$ are not presented in Fig. 1b. The best fit to the $(\mathrm{O} / \mathrm{H})_{P}$ data is

$12+\log (\mathrm{O} / \mathrm{H})_{P}=8.76( \pm 0.08)-0.024( \pm 0.005) R_{\mathrm{G}}$.

This $(\mathrm{O} / \mathrm{H})_{P}-R_{\mathrm{G}}$ relation is shown by the dashed line in the Fig. 1b. The mean residual from the $(\mathrm{O} / \mathrm{H})_{P}-R_{\mathrm{G}}$ relation is $0.084 \mathrm{dex}$. The corresponding error in the slope is $\pm 0.005 \mathrm{dex} / \mathrm{kpc}$, i.e. the same as in the case of gradient traced by the $(\mathrm{O} / \mathrm{H})_{T_{\mathrm{e}}}$ abundances. Inspection of Fig. $1 \mathrm{~b}$ shows that the radial $(\mathrm{O} / \mathrm{H})_{P}$ abundance distribution is in agreement with the $(\mathrm{O} / \mathrm{H})_{T_{\mathrm{e}}}$ distribution.

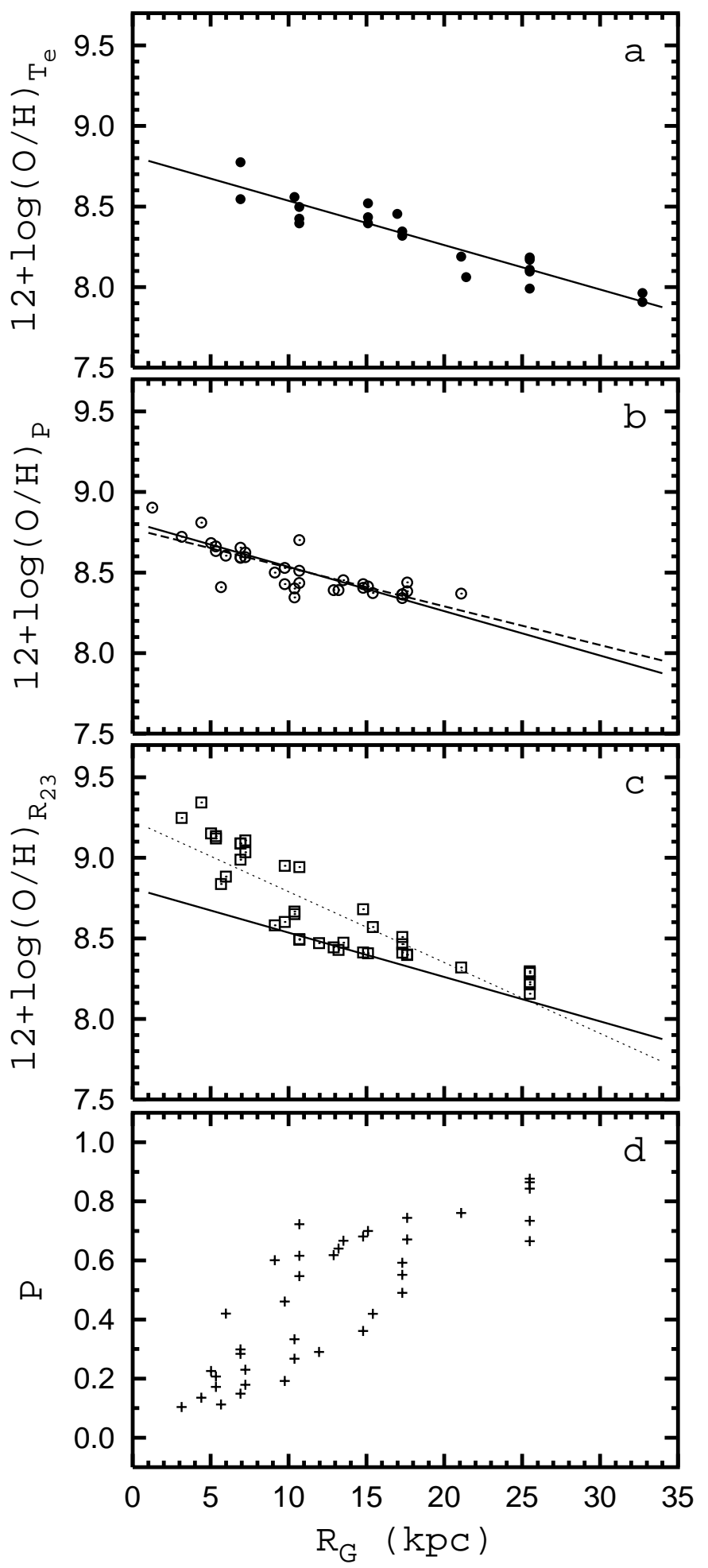

Fig. 1. Gradients in the properties of $\mathrm{M}$ 101. a) The $(\mathrm{O} / \mathrm{H})_{T_{\mathrm{e}}}$ abundance distribution (filled circles) and the best fit (solid line). b) The $(\mathrm{O} / \mathrm{H})_{P}$ abundance distribution (open circles) and the best fit (dashed line). The solid line is the $(\mathrm{O} / \mathrm{H})_{T_{\mathrm{e}}}-$ $R_{\mathrm{G}}$ relation. c) The $(\mathrm{O} / \mathrm{H})_{R_{23}}$ abundance distribution (open squares) and the best fit (dotted line). The solid line is the $(\mathrm{O} / \mathrm{H})_{T_{\mathrm{e}}}-R_{\mathrm{G}}$ relation. d) The excitation parameter $P$ as a function of galactocentric distance. 
Figure 1c shows the radial $(\mathrm{O} / \mathrm{H})_{R_{23}}$ abundance distribution (the open squares) for the same $\mathrm{H}$ II regions as in Fig. 1b. The best fit to the $(\mathrm{O} / \mathrm{H})_{R_{23}}$ data is

$$
12+\log (\mathrm{O} / \mathrm{H})_{R_{23}}=9.23( \pm 0.16)-0.044( \pm 0.010) R_{\mathrm{G}} \text {. (4) }
$$

This $(\mathrm{O} / \mathrm{H})_{R_{23}}-R_{\mathrm{G}}$ relation is shown by the dotted line in Fig. 1c. The mean residual from the $(\mathrm{O} / \mathrm{H})_{R_{23}}-R_{\mathrm{G}}$ relation is $0.16 \mathrm{dex}$. The corresponding error in the slope is $\pm 0.010 \mathrm{dex} / \mathrm{kpc}$. Inspection of Fig. 1c shows that the $(\mathrm{O} / \mathrm{H})_{R_{23}}$ abundances in $\mathrm{H}$ II regions with large galactocentric distances are close to the $(\mathrm{O} / \mathrm{H})_{T_{\mathrm{e}}}-R_{\mathrm{G}}$ relation, while the $(\mathrm{O} / \mathrm{H})_{R_{23}}$ abundances in $\mathrm{H}$ II regions in the inner part of $\mathrm{M} 101$ have significant deviations from the $(\mathrm{O} / \mathrm{H})_{T_{\mathrm{e}}}-R_{\mathrm{G}}$ relation. This behaviour of $(\mathrm{O} / \mathrm{H})_{R_{23}}$ abundances with galactocentric distance confirms the conclusion of Paper II that the $R_{23}$ calibration of Edmunds \& Pagel (1984) provides a realistic oxygen abundances in high-excitation $\mathrm{H}$ II regions, but overestimates them in low-excitation ones. Indeed, the $\mathrm{H}$ II regions with galactocentric distances larger than around $17 \mathrm{kpc}$ are high- and moderate-excitation $(P$ values are higher than around 0.6$)$ ones, Fig. 1d, their $(\mathrm{O} / \mathrm{H})_{R_{23}}$ abundances are close to the $(\mathrm{O} / \mathrm{H})_{T_{\mathrm{e}}}-R_{\mathrm{G}}$ relation. By contrast, the $\mathrm{H}$ II regions with galactocentric distances smaller than around $9 \mathrm{kpc}$ are low-excitation $(P$ values are less than around 0.3$)$ ones, Fig. 1d, their $(\mathrm{O} / \mathrm{H})_{R_{23}}$ abundances have significant deviations from the $(\mathrm{O} / \mathrm{H})_{T_{\mathrm{e}}}-R_{\mathrm{G}}$ relation.

The form of oxygen abundance gradient in M 101 has been investigated in a number of studies (Evans 1986; Vila-Costas \& Edmunds 1992; Henry \& Howard 1995; Kennicutt \& Garnett 1996). It has been noted by Kennicutt \& Garnett (1996) and Roy \& Walsh (1997) that the shape of the $(\mathrm{O} / \mathrm{H})_{R_{23}}$ distribution is very sensitive to the precise form of the $R_{23}$ calibration. In the case of M 101 Kennicutt \& Garnett (1996) have found that the Edmunds \& Pagel (1984) calibration produces a "steep-shallow" break in the slope of the distribution (see also Vila-Costas \& Edmunds 1992), while the Dopita \& Evans (1986) and McCall et al. (1985) calibrations yield distributions which are very well fitted with a single exponential function, though the slope of the exponential differs considerably between the two calibrations. A careful examination of Fig. 1 suggests that the distributions of the $(\mathrm{O} / \mathrm{H})_{T_{\mathrm{e}}}$, Fig. 1a, and $(\mathrm{O} / \mathrm{H})_{P}$ abundances, Fig. 1b, can be fitted quite comfortably within the scatter by the same exponential with constant slope. There is hint of a turnover in the slope of the $(\mathrm{O} / \mathrm{H})_{R_{23}}$ abundance distribution, Fig. 1c. This turnover is artifact and reflects the increase of error in $(\mathrm{O} / \mathrm{H})_{R_{23}}$ abundance with decrease of galactocentric distance caused by variations in excitation parameter, Fig. 1d.

An important result of the present study is the rather low value of the central oxygen abundance in M 101. This is in agreement with the result of Kinkel \& Rosa (1994), who showed the need to lowering all $\mathrm{H}$ II region abundances obtained on the basis of the $R_{23}$ calibration after Edmunds \& Pagel (1984) by at least 0.2 at intrinsic solar like $\mathrm{O} / \mathrm{H}$ values and above. One more large spiral galaxy in which the $(\mathrm{O} / \mathrm{H})_{T_{\mathrm{e}}}$ abundance distribution has been established is the Milky Way Galaxy. Caplan et al. (2000) and Deharveng et al. (2000) have analysed Galactic $\mathrm{H}$ II regions and have obtained the slope $-0.0395 \mathrm{dex} / \mathrm{kpc}$ with central oxygen abundance $12+\log (\mathrm{O} / \mathrm{H})=8.82$, close to the solar value $12+\log (\mathrm{O} / \mathrm{H})_{\odot}=8.83$ (Grevesse \& Sauval 1998), and $12+\log (\mathrm{O} / \mathrm{H})=8.48$ at the solar galactocentric distance, around a factor of 2 lower than the solar abundance. Rodriques (1999) has considered seven bright Galactic H II regions with galactocentric distances in the range $6-10 \mathrm{kpc}$ and has found that all the $\mathrm{HII}$ regions studied are characterized by similar abundances, $12+\log (\mathrm{O} / \mathrm{H}) \sim 8.45 \pm 0.1$. This value of the oxygen abundance at the solar radius is close to the value of the interstellar oxygen in the vicinity of the Sun which is about two-thirds of the solar oxygen abundance (Meyer et al. 1998). Those data taken together suggest that the oxygen abundance at the solar radius is $1 / 2 \div 2 / 3$ of the solar oxygen abundance and increases up to about solar value in the centre of our Galaxy. The central oxygen abundance in $\mathrm{M} 101$ based on the $(\mathrm{O} / \mathrm{H})_{T_{\mathrm{e}}}\left(\right.$ or $\left.(\mathrm{O} / \mathrm{H})_{P}\right)$ abundances is close to that in our Galaxy.

Thus, the consideration of M 101 suggests the following. The available $(\mathrm{O} / \mathrm{H})_{T_{\mathrm{e}}}$ abundances allow to establish quite firmly the parameters of the radial oxygen abundance distribution (the central oxygen abundance and the gradient) within $\mathrm{M} 101$. The parameters of the $(\mathrm{O} / \mathrm{H})_{P}$ abundance distribution are close to those of the $(\mathrm{O} / \mathrm{H})_{T_{\mathrm{e}}}$ abundance distribution. The parameters of the $(\mathrm{O} / \mathrm{H})_{R_{23}}$ abundance distribution differ significantly from those of the $(\mathrm{O} / \mathrm{H})_{T_{\mathrm{e}}}$ abundance distribution: the values of the gradient and the central oxygen abundance based on the $(\mathrm{O} / \mathrm{H})_{R_{23}}$ data are overestimated as compared to values derived from the $(\mathrm{O} / \mathrm{H})_{T_{\mathrm{e}}}$ distribution.

\subsection{The dispersion in abundance}

The dispersion in $(\mathrm{O} / \mathrm{H})_{P}$ abundance at fixed radius is coincident with that in $(\mathrm{O} / \mathrm{H})_{T_{\mathrm{e}}}$ abundance, $\sim 0.08$ dex. In the general case, the error in line intensity measurements can make contribution to the scatter at fixed galactocentric distance. The precision of present-day determinations of the oxygen abundances in high-metallicity HiI regions through the $T_{\mathrm{e}}$-method seems to be about 0.1 dex (Deharveng et al. 2000). The precision of oxygen abundance determination with the $P$-method is comparable to that of the $T_{\mathrm{e}}-$ method (Paper II). Those facts taken together suggest none, or only marginal, scatter at a given galatocentric distance. However, Kennicutt \& Garnett (1996) advocate that the quality of their specta is sufficiently high that observational errors contribute negligibly to the dispersion. If this is the case it cannot be excluded that some part of the dispersion in $(\mathrm{O} / \mathrm{H})_{P}$ abundance reflects the actual deviations of oxygen abundances in individual $\mathrm{H}$ II regions from the general $(\mathrm{O} / \mathrm{H})_{P}-R_{\mathrm{G}}$ trend. 


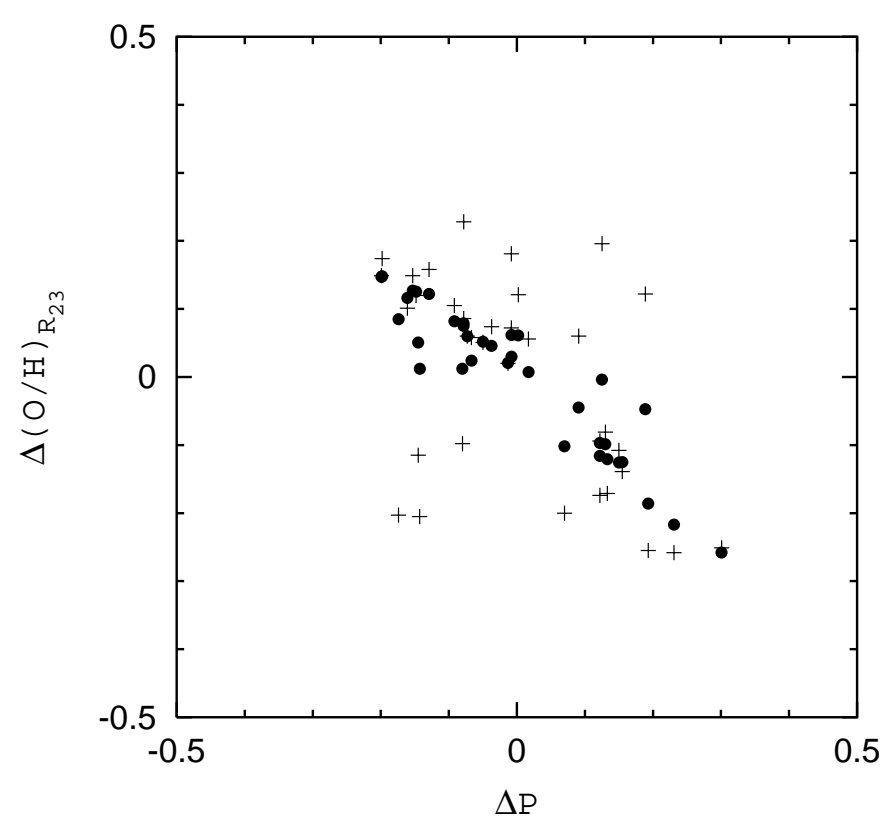

Fig. 2. The $\log (\mathrm{O} / \mathrm{H})_{R_{23}}$ residual from a mean linear fit, $\Delta(\mathrm{O} / \mathrm{H})_{R_{23}}$, as a function of excitation parameter $P$ residual, $\Delta P$, for H II regions from Kennicutt \& Garnett (1996). The data obtained with original $(\mathrm{O} / \mathrm{H})_{R_{23}}$ are shown by pluses, the data obtained with corrected $(\mathrm{O} / \mathrm{H})_{R_{23}}$ are shown by filled circles.

The dispersion in $(\mathrm{O} / \mathrm{H})_{R_{23}}$ abundance at fixed radius, $\sim 0.16$ dex, is appreciable larger than that in $(\mathrm{O} / \mathrm{H})_{P}$ abundance, $\sim 0.08$ dex. The following interpretation of this fact can be suggested. The extra dispersion in $(\mathrm{O} / \mathrm{H})_{R_{23}}$ abundance at fixed radius is an artifact and reflects the dispersion in excitation parameter $P$.

Kennicutt \& Garnett (1996) have also suggested that the dispersion in $(\mathrm{O} / \mathrm{H})_{R_{23}}$ abundance at fixed radius can be attributed partly to variations in excitation parameter, but they did not find a firm confirmation of this suggestion. Following the strategy of Kennicutt and Garnett we fitted the radial variations in $\log (\mathrm{O} / \mathrm{H})_{R_{23}}$ and $P$ by linear relations and considered correlation between the residuals in $\log (\mathrm{O} / \mathrm{H})_{R_{23}}$ and $P$. This correlation is shown in Fig. 2 by pluses. As can seen in Fig. 2 the correlation between the residuals is very weak, if at all. Why? We will demonstrate that this is due to two reasons; i) abundance dispersion in the $\mathrm{H}$ II regions themselves, and $i$ ) a feature of the sample.

Figure 3 shows $\delta(\mathrm{O} / \mathrm{H})_{R_{23}}$ (the deviation of individual $\log (\mathrm{O} / \mathrm{H})_{R_{23}}$ from $\log (\mathrm{O} / \mathrm{H})_{P}-R_{\mathrm{G}}$ fit, Eq. (3)) versus $\Delta(\mathrm{O} / \mathrm{H})_{P}\left(\right.$ the $\log (\mathrm{O} / \mathrm{H})_{P}$ residual from the same fit) for H II regions from Kennicutt \& Garnett (1996). The triangles are $\mathrm{H}$ II regions with $P>0.5$, the pluses are $\mathrm{H}$ II regions $0.5>P>0.3$, the circles are those with $0.3>P$. Figure 3 shows that the variations in excitation parameter $P$ (from $\sim 0.9$ to $\sim 0.1$, see Fig. $1 \mathrm{~d}$ ) result in the difference in $(\mathrm{O} / \mathrm{H})_{R_{23}}$ as large as $\sim 0.6$ dex. Figure 3 shows that in the general case the $\delta(\mathrm{O} / \mathrm{H})_{R_{23}}$ is the sum of two parts; the first is the $\Delta(\mathrm{O} / \mathrm{H})_{P}$ and the second is the deviation depending on the value of excitation parameter $P$.

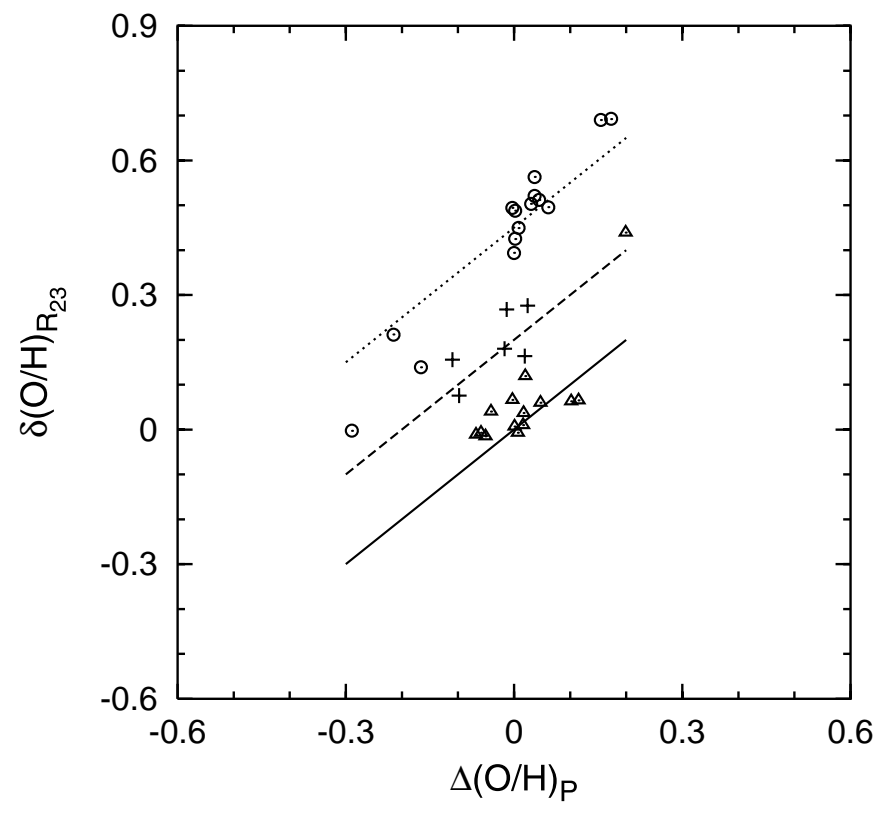

Fig. 3. The $\delta(\mathrm{O} / \mathrm{H})_{R_{23}}$ is the deviation of $\log (\mathrm{O} / \mathrm{H})_{R_{23}}$ from the $\log (\mathrm{O} / \mathrm{H})_{P}-R_{\mathrm{G}}$ fit. The $\Delta(\mathrm{O} / \mathrm{H})_{P}$ is the $\log (\mathrm{O} / \mathrm{H})_{P}$ residual from the same fit. The triangles are $\mathrm{HII}$ regions with $P>0.5$, the pluses are $\mathrm{H}$ II regions $0.5>P>0.3$, the circles are those with $0.3>P$. The solid line is the relation $\delta(\mathrm{O} / \mathrm{H})_{R_{23}}=\Delta(\mathrm{O} / \mathrm{H})_{P}$, the dashed line is the relation $\delta(\mathrm{O} / \mathrm{H})_{R_{23}}=\Delta(\mathrm{O} / \mathrm{H})_{P}+0.2$, the dotted line is the relation $\delta(\mathrm{O} / \mathrm{H})_{R_{23}}=\Delta(\mathrm{O} / \mathrm{H})_{P}+0.45$.

The relevant feature of the sample of $\mathrm{H}$ II regions of M 101 from Kennicutt \& Garnett (1996) can be seen in Fig. 1d; the H II regions occupy a relatively narrow band in the $P-R_{\mathrm{G}}$ diagram. Due to this feature of the sample of $\mathrm{H}$ II regions the variations in excitation parameter $P$ at fixed galactocentric distance do not exceed $\sim 0.4$, or the maximum deviation of excitation parameter from mean value is around \pm 0.2 . The maximum deviation around \pm 0.15 dex in $(\mathrm{O} / \mathrm{H})_{R_{23}}$ corresponds to this maximum deviation of excitation parameter. Thus, the expected maximum deviation in $(\mathrm{O} / \mathrm{H})_{R_{23}}$ due to the deviation of excitation parameter from mean value is only twice the average actual deviation of oxygen abundances in individual H II regions from general $(\mathrm{O} / \mathrm{H})_{P}-R_{\mathrm{G}}$ trend, that can mask the correlation between the residuals in $\log (\mathrm{O} / \mathrm{H})_{R_{23}}$ and $P$. Therefore the $(\mathrm{O} / \mathrm{H})_{R_{23}}$ values were corrected for deviations caused by dispersion in the $\mathrm{H}$ II regions themselves

$(\mathrm{O} / \mathrm{H})_{R_{23}}^{\mathrm{c}}=(\mathrm{O} / \mathrm{H})_{R_{23}}-\Delta(\mathrm{O} / \mathrm{H})_{P}$.

The $\Delta P-\Delta(\mathrm{O} / \mathrm{H})_{R_{23}}^{\mathrm{c}}$ diagram for corrected $(\mathrm{O} / \mathrm{H})_{R_{23}}^{\mathrm{c}}$ values has been constructed. This diagram is shown in Fig. 2 by filled circles. As can seen in Fig. 2 the correlation between the residuals is rather tight in this case. This is reliable confirmation that the dispersion in $(\mathrm{O} / \mathrm{H})_{R_{23}}$ abundance at fixed radius is caused partly by variations in excitation parameter.

As for the feature of Kennicutt and Garnett's sample of $\mathrm{H}$ II regions, the lack of high-excitation H II regions in central part of M 101 can be explained by the 


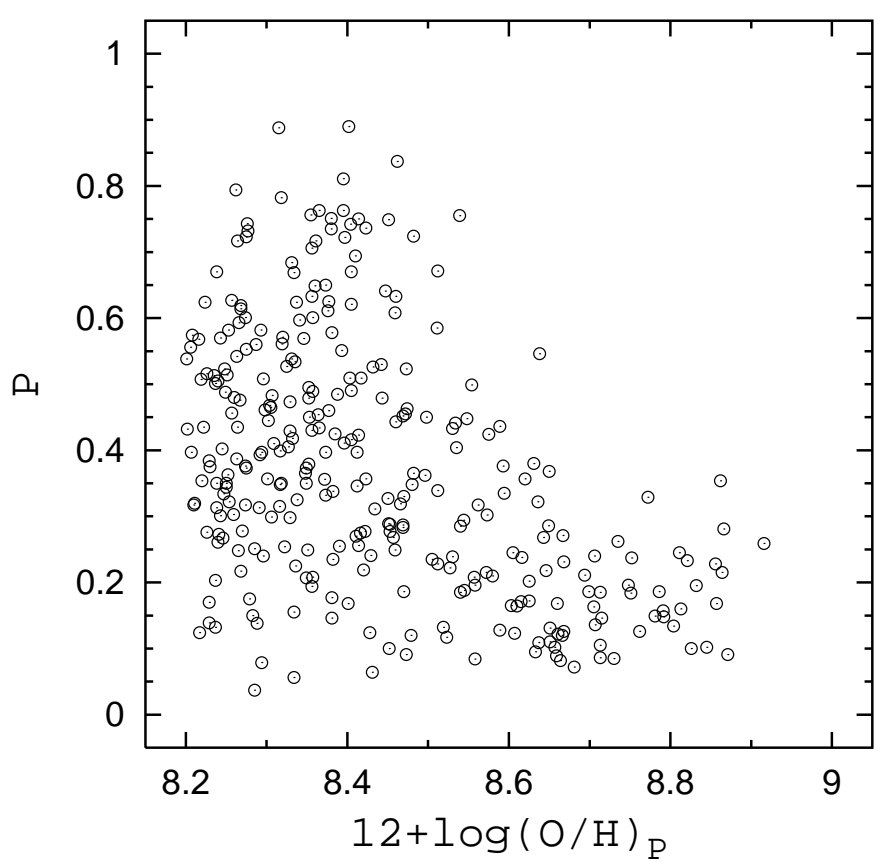

Fig. 4. The $P-(\mathrm{O} / \mathrm{H})_{P}$ diagram for $\mathrm{H}$ II regions from Zaritsky et al. (1994) and van Zee et al. (1998).

ionization temperature gradient. The radial distribution of the ionization temperature for the $\mathrm{H}$ II regions in M 101 has been investigated by Vilchez \& Pagel (1988). They showed that there is a clear gradient in the temperature along the disk of M 101. The data for other galaxies confirm the softening of the ionizing spectra with increasing metal abundance (Kennicutt et al. 2000, and references therein). Figure 4 shows the $P-(\mathrm{O} / \mathrm{H})_{P}$ diagram for a large sample of $\mathrm{H}$ II regions from Zaritsky et al. (1994) and van Zee et al. (1998). Examination of Fig. 4 shows that the maximum value of the excitation parameter $P$ is dependent on the metallicity. Since excitation parameter $P$ is an indicator of hardness of the ionizing radiation, the $P-(\mathrm{O} / \mathrm{H})_{P}$ diagram for large sample of $\mathrm{H}$ II regions confirms the softening of the ionizing spectra with increasing metal abundance. But the lack of low-excitation $\mathrm{H}$ II at outer part of M 101 seems to be conditioned by selection.

Kennicutt \& Garnett (1996) concluded that there is hint that some of the dispersion in $(\mathrm{O} / \mathrm{H})_{R_{23}}$ abundances in $\mathrm{M} 101$ is the result of a large-scale deviation from azimuthal symmetry. Is there spatial asymmetry in $(\mathrm{O} / \mathrm{H})_{P}$ abundance residuals? Figure 5 shows the spatial distribution of $(\mathrm{O} / \mathrm{H})_{P}$ abundance residuals. Pluses are $\mathrm{HII}$ regions with $-0.08<\Delta(\mathrm{O} / \mathrm{H})_{P}<0.08$, filled circles are those with $\Delta(\mathrm{O} / \mathrm{H})_{P}>0.08$, open circles are those with $\Delta(\mathrm{O} / \mathrm{H})_{P}<-0.08$. Indeed, some asymmetry in the spatial distribution of $(\mathrm{O} / \mathrm{H})_{P}$ abundance residuals can be seen in Fig. 5. However, following Kennicutt \& Garnett (1996) we can conclude that more data are needed to test whether this asymmetry is real.

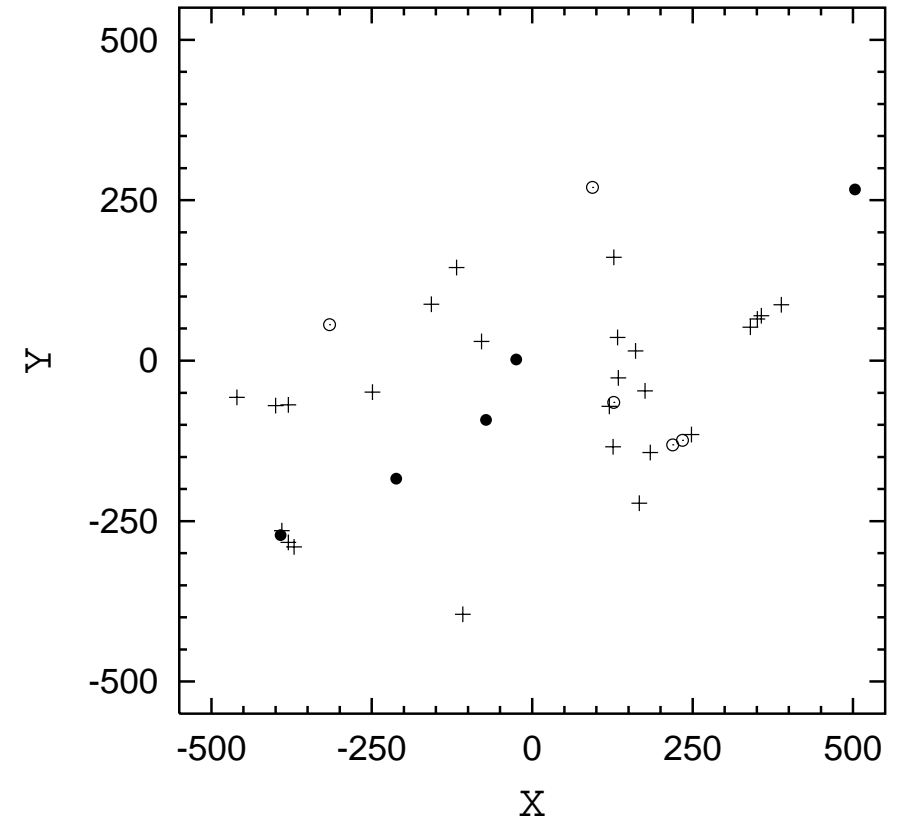

Fig. 5. The spatial distribution of the abundance residuals. Pluses are $\mathrm{H}$ II regions with $-0.08<\Delta(\mathrm{O} / \mathrm{H})_{P}<0.08$, filled circles are those with $\Delta(\mathrm{O} / \mathrm{H})_{P}>0.08$, open circles are those with $\Delta(\mathrm{O} / \mathrm{H})_{P}<-0.08$.

\section{Conclusions}

The radial distributions of the oxygen abundances determined in three different ways (with the classic $T_{\mathrm{e}^{-}}$ method, with the $R_{23}$-method, and with the $P$-method) in H II regions of large spiral galaxy M 101 have been compared.

It has been found that the available $(\mathrm{O} / \mathrm{H})_{T_{\mathrm{e}}}$ abundances are sufficient in quantity and quality for an accurate determination of the parameters of the radial abundance distribution (the central oxygen abundance and the gradient). We found that $12+\log (\mathrm{O} / \mathrm{H})_{T_{\mathrm{e}}}=8.81( \pm 0.08)-$ $0.028( \pm 0.005) R_{\mathrm{G}}(\mathrm{kpc})$.

It has been found that the parameters of the radial $(\mathrm{O} / \mathrm{H})_{P}$ abundance distribution are close to those of the $(\mathrm{O} / \mathrm{H})_{T_{\mathrm{e}}}$ abundance distribution. We obtained that $12+\log (\mathrm{O} / \mathrm{H})_{P}=8.76( \pm 0.08)-0.024( \pm 0.005) R_{\mathrm{G}}(\mathrm{kpc})$. This confirms the conclusion of Paper II that the $(\mathrm{O} / \mathrm{H})_{P}$ abundances are as credible as the $(\mathrm{O} / \mathrm{H})_{T_{\mathrm{e}}}$ abundances.

It has been obtained that the parameters of the radial $\mathrm{O} / \mathrm{H}_{R_{23}}$ abundance distribution differ significantly from those of the $\mathrm{O} / \mathrm{H}_{T_{\mathrm{e}}}$ abundance distribution. We found that $12+\log (\mathrm{O} / \mathrm{H})_{R_{23}}=9.23( \pm 0.16)-0.044( \pm 0.010) R_{\mathrm{G}}(\mathrm{kpc})$. This confirms our speculation that the central oxygen abundance and gradient slope based on the $(\mathrm{O} / \mathrm{H})_{R_{23}}$ data can be appreciably overestimated.

The dispersion in $(\mathrm{O} / \mathrm{H})_{R_{23}}$ abundance at fixed radius is appreciable larger than that in $(\mathrm{O} / \mathrm{H})_{P}$ abundance. It has been demonstrated that the extra dispersion in $(\mathrm{O} / \mathrm{H})_{R_{23}}$ abundance is an artifact and reflects the dispersion in excitation parameter $P$. 
Acknowledgements. It is a pleasure to thank J. M. Vilchez and D. R. Garnett for their helpful comments on this work. I thank the referee, Prof. B. E. J. Pagel, for helpful comments and suggestions as well as improving the English text. This study was partly supported by the NATO grant PST.CLG.976036 and the Joint Research Project between Eastern Europe and Switzerland (SCOPE) No. 7UKPJ62178.

\section{References}

Alloin, D., Collin-Souffrin, S., Joly, M., \& Vigrough, L. 1979, A\&A, 78, 200

Caplan, J., Deharveng, L., Pena, M., Costero, R., \& Blondel, C. 2000, MNRAS, 311, 317

Deharveng, L., Pena, M., Caplan, J., \& Costero, R. 2000, MNRAS, 311, 329

Dopita, M. A., \& Evans, I. N. 1986, ApJ, 307, 431

Edmunds, M. G., \& Pagel, B. E. J. 1984, MNRAS, 211, 507

Evans, I. N. 1986, ApJ, 309, 544

Garnett, D. R. 1992, AJ, 103, 1330

Garnett, D. R., \& Kennicutt, R. C. 1994, ApJ, 426, 123

Garnett, D. R., Shields, G. A., Peimbert, M., et al. 1999, ApJ, 513,168

Grevesse, N., \& Sauval, A. J. 1998, in Solar composition and its evolution - from core to corona, ed. G. Fronlich, M. C. E. Huber, S. K. Solanki, \& R. von Steiger (Kluwer, Space Sciences Ser. of ISSI, vol. 5), 161

Henry, R. B. C., \& Howard, J. W. 1995, ApJ, 438, 170
Kennicutt, R. C., Bresolin, F., French, H., \& Martin, P. 2000, ApJ, 537, 589

Kennicutt, R. C., \& Garnett, D. R. 1996, ApJ, 456, 504

Kinkel, U., \& Rosa, M. R. 1994, A\&A, 282, L37

McCall, M. L., Rybski, P. M., \& Shields, G. A. 1985, ApJS, 57,1

Meyer, D. M., Jura, M., \& Cardelli, J. A. 1998, ApJ, 493, 222

Pagel, B. E. J., Edmunds, M. G., Blackwell, D. E., Chun, M. S., \& Smith, G. 1979, MNRAS, 189, 95

Pagel, B. E. J., Simonson, E. A., Terlevich, R. J., \& Edmunds, M. G. 1992, MNRAS, 255, 325

Pilyugin, L. S. 2000, A\&A, 362, 325, Paper I

Pilyugin, L. S. 2001, A\&A, 369, 594, Paper II

Rayo, J. F., Peimbert, M., \& Torres-Peimbert, S. 1982, ApJ, 255,1

Rodriguez, M. 1999, A\&A, 351, 1075

Roy, J.-R., \& Walsh, J. R. 1997, MNRAS, 288, 715

Searle, L. 1971, ApJ, 168, 327

Shields, G. A., \& Searle, L. 1978, ApJ, 222, 821

Smith, H. E. 1975, ApJ, 199, 591

Torres-Peimbert, S., Peimbert, M., \& Fierro, J. 1989, ApJ, 345, 186

van Zee, L., Salzer, J. J., Haynes, M. P., O’Donoghue, A. A., \& Balonek, T. J. 1998, AJ, 116, 2805

Vila-Costas, M. B., \& Edmunds, M. G. 1992, MNRAS, 259, 121

Vilchez, J. M., \& Pagel, B. E. J. 1988, MNRAS, 231, 257

Zaritsky, D. 1992, ApJ, 390, L73

Zaritsky, D., Kennicutt, R. C., \& Huchra, J. P. 1994, ApJ, 420, 87 